\title{
EFFECT OF MOBILITY MODELS ON REINFORCEMENT LEARNING BASED ROUTING ALGORITHM APPLIED FOR SCALABLE AD HOC NETWORK ENVIRONMENT
}

\author{
Shrirang.Ambaji.Kulkarni ${ }^{1}$ and G.Raghavendra Rao ${ }^{2}$ \\ Department of Computer Engineering, National Institute of Engineering, Mysore, India \\ sakulkarniegit.edu ${ }^{1}$ and grrao56@gmail. $\mathrm{com}^{2}$
}

\begin{abstract}
Mobile Ad Hoc Network faces the greatest challenge for better performances in terms of mobility characterization. The mobility of nodes and their underlying mobility models have a profound effect on the performances of routing protocols which are central to the design of ad hoc networks. Most of the traditional routing algorithms proposed for ad hoc networks do not scale well when the traffic variation increases drastically. To model a solution to this problem we consider a reinforcement learning based routing algorithm for ad hoc network known as SAMPLE. Most the scalability issues for ad hoc network performance investigation have not considered the group mobility of nodes. In this paper we model realistic group vehicular mobility model and analyze the robustness of a reinforcement learning based routing algorithm under scalable conditions.
\end{abstract}

\section{KEYWORDS}

Routing protocols, mobility models, scalability and reinforcement learning.

\section{INTRODUCTION}

A mobile ad hoc network (MANET) is a group of mobile nodes which form an autonomous system and are connected via wireless links to form a communication network [1]. A mobile ad hoc network exhibits special set of characteristics like dynamic topologies, variable wireless link capacity, limited energy resources and security services [2]. To achieve scalable performances with respect to the above characteristics is one of the most important challenges of ad hoc networks. To mimic the realistic movement of nodes in real world we visualize in terms of mobility models. The mobility characteristics like speed, direction and its correlation with other nodes leads to a major impact on the routing protocols which are central to the design of ad hoc networks [3]. Many mobility models have been proposed in the study of ad hoc networks such as Random Waypoint Mobility Model [4],[5],[27],[28] Steady State Random Waypoint [6],[29], Gauss Markov Model [30],[31], Reference Point Group Mobility Model [25], Manhattan Mobility Model [7],[8] and City Section Mobility Model [9]. In this paper we propose to study the impact of these mobility models under scalable conditions by analyzing with appropriate 
mobility metrics. Most of the research in the direction of mobility models has been to characterize the mobility of nodes individually. In this paper we extend the characterization of node mobility for groups and propose a new realistic group vehicular mobility model for scalable ad hoc network operations. For any form of network to be popular, it must exhibit scale economies. Thus the average cost per user of the network declines as the number of users or host computer increases [10]. A scalable application for mobile ad hoc network can be visualized as hundreds of vehicles interacting with each other in a decentralized manner and moving across pathways or lanes in cities [11]. The unique characteristics of mobile ad hoc networks impose a great challenge in their practical realization. Most of the traditional routing algorithms for ad hoc networks like Destination-Sequenced Distance Vector (DSDV) [12], Ad- Hoc On-demand Distance Vector (AODV) [13], [14] and Dynamic Source Routing (DSR) [15] do not perform well when tested under scalable conditions. To meet the challenges of routing in unpredictable dynamic environments, we consider routing techniques based on reinforcement learning. Reinforcement learning is an interesting methodology with application to routing in dynamic networks. Here an autonomous agent takes actions in an environment and receives reward or penalty for the same [16]. After a set of trial and error runs it learns the optimal policy and in the process maximizes the total reward. Thus we consider one of the routing algorithms based on reinforcement learning known as SAMPLE [17], [39]. SAMPLE is a routing protocol which belongs to the class of on-demand routing protocols and uses collaborative reinforcement learning (CRL), whereby agents interact with one another cooperatively to solve a distributed optimization problem. To aid the routing protocols in this complex interplay of scalable mobile nodes forming dynamic topologies, we propose a realistic group vehicular mobility model. The proposed mobility model characterizes group mobility rather than mobility of individual nodes and is based on movement of nodes along maps drawn from TIGER (Topologically Integrated Geographic Encoding and Referencing) [18].

In Section 2 we discuss the related work and the motivation for our work. In Section 3 we consider the various mobility models and analyze their impact under scalable conditions with appropriate mobility metrics. Section 4 discusses the routing protocols considered for application in scalable environments. In Section 5 we discuss the performance of routing protocols AODV and SAMPLE for scalable environment under group mobility models and the proposed realistic model. Section 6 concludes the paper.

\section{RELATED WORK}

P Nurmi [19] in his work on applying reinforcement learning algorithms for ad hoc networks modeled the problem where routing decisions of nodes not only depended on selfishness but also on the energy level of nodes. In the simulation based investigation he considered only Random Waypoint Mobility Model with 100 nodes. J Dowling et.al [17] applied collaborative reinforcement learning to enable agents to apply reinforcement learning to solve optimization problems in ad hoc networks. They considered only Random Waypoint Mobility Model with 
constant bit rate traffic of 4 packets per second. They did not consider group mobility model for node mobility. V Naumov and T Gross [21] in their analysis of scalability of routing methods for ad hoc networks investigated the performance of two routing protocols namely AODV and DSR. They analyzed the limiting factor in terms of scalability of DSR caused by aggressive use of cached information resulting in high number of administrative packets. They also concluded that both the protocols showed poor performance in terms of high traffic load. They considered only Random Waypoint Mobility Model. This served as a motivation to consider reinforcement learning algorithm under scalable condition and consider the impact of scalability on different mobility models. B J Kwak et.al. [20] in their work on scalability of ad hoc networks analyzed the packet traffic at the center of the network. They concluded through their work that the packet traffic at the center of the network was linearly related to the network size. F Bai et.al [22] developed a framework IMPORTANT to evaluate the impact of mobility models on the performance of routing protocols for MANET. Their results proved that routing protocol performance vary across various mobility models. They developed suitable mobility metrics to measure the impact of mobility models on routing protocols for MANET. Zhou B et.al. [23] proposed a real track mobility model derived from the virtual track mobility model [24]. The virtual track models the car movements along a graph where vertices are defined as switch stations and edges as virtual tracks. Here nodes move in groups according to the Reference point group mobility model (RPGM) [25]. The real track model applies the virtual track to real road topologies. The goal of real track model is to mimic clustering of vehicles at intersections. In our proposed mobility model we take the group characterization ahead and apply it to realistic maps to model a scalable application of MANET and aid a reinforcement learning routing algorithm to perform optimally under varying traffic and scalable ad hoc network environment.

\section{MobILITy Models}

Mobility models are crucial for simulation based performance analysis of routing protocols as they capture the movements of real mobile nodes [26]. The mobility models we consider for realizations of scalable conditions are Steady State Random Waypoint Mobility Model (SRWMM), Gauss Markov Model (GMM), Reference Point Group Mobility Model (RPGM), Manhattan Mobility Model (MM) and City Section Mobility Model (CSMM). We also propose a novel Realistic Optimized Group Vehicular Mobility Model (ROPGVMM)

\subsection{Realistic Optimized Group Vehicular Mobility Model}

Most of the research in mobility models has been in the individual characterization of mobile nodes rather than as a group of nodes. One of the short comings of the City Section Mobility Model is that the streets in the City Section are created by users and hence is only as realistic as user's imaginations. Thus we propose a mobility models that takes the best features of group mobility and applies to vehicular motion of mobile nodes under realistic road conditions i.e. on a realistic map of a US county. The Realistic Optimized Group Vehicular Mobility (ROPGVM) is 
an attempt to provide a mobility model for realistic scalable applications for MANET and also for optimal performances of routing protocols. The realistic map of an important US county is taken from the TIGER database [18] and converted to a model for simulation [32], [33]. The realistic map is illustrated in Figure 1.

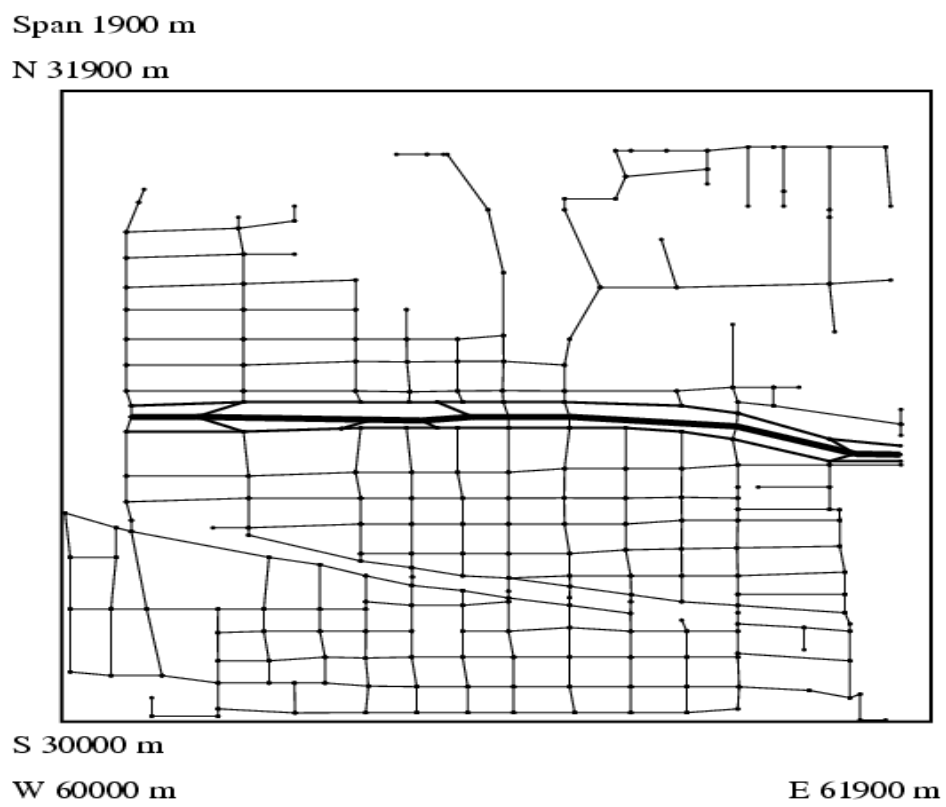

Figure 1. A Realistic map depicting a street scenario for 1900 x 1900 region.

The algorithm for the proposed mobility model is as follows.

Algorithm: Realistic Optimized Group Vehicular Mobility Model

Input: Number of Nodes $(\mathrm{N})$, Number of Groups $(\mathrm{G})$, Speed Deviation and Angle Deviation (A)

1. Read the input parameters like Nodes $(\mathrm{N})$ and number of Groups $(\mathrm{G})$

2. Read the Speed Deviation and Angle Deviation by which individual nodes deviate from their group leader.

3. Set the movement of the group leader at time $t$ to the motion vector Vtgroup defined by our vehicular trace file, 4 trace files for each scenario and for each group leader derived from a realistic map defining the pathways for vehicular movement

4. The speed and direction of each group member deviating from its leader is given by

$\mathrm{Vm}(\mathrm{t})=\mathrm{Vl}(\mathrm{t})+\operatorname{random}() * \mathrm{SDR} * \max \_$speed

$\theta \mathrm{m}(\mathrm{t})=\theta 1(\mathrm{t})+\operatorname{random}() * A D R *$ max_angle.

where SDR is speed deviation ratio and ADR is angle deviation ratio

max_speed $\{10,20,30,40,50,60\}$ and max_angle $\{0,2 \Pi\}$ in our experiment

5. Capture the movements of nodes with all the details in a trace file for NS-2 simulator. 


\subsection{Mobility Metrics}

We have considered the following mobility metrics from [22] for our analysis of mobility models.

- Average Link Duration: - This metric specifies the longest interval of time [t1, $t 2]$ for nodes $i$ and $\mathrm{j}$ forming the link $(\mathrm{i}, \mathrm{j}$ ). This is then averaged for all node pairs for all existing links specifying the Equation 4

$$
L D^{\prime}=\frac{\sum_{t=0}^{T} \sum_{i=1}^{N} \Sigma_{j=i+1}^{N} L D(i, j, t 1)}{F}
$$

where $P$ is no of tuples $(i, j, t 1)$ and $L D(i, j, t 1) \neq 0$

- $\quad$ Average Relative Speed:- Relative speed is given by Equation 5 .

$\operatorname{RST}(i, j, t)=|\operatorname{Vi}(\mathrm{t})-\operatorname{Vj}(\mathrm{t})|$

where $\operatorname{Vi}(t)$ and $\operatorname{Vj}(t)$ is the velocity vector of node $i$ and $j$ at time t. The average value of $\operatorname{RST}(i, j, t)$ is given by Equation 6 .

$$
\operatorname{RST}^{*}=\frac{\sum_{i=1}^{N} \sum_{j=1}^{N} \Sigma_{t=1}^{T} R S T(i, j, t)}{P}
$$

where $\mathrm{P}$ is no of tuples $(\mathrm{i}, \mathrm{j}, \mathrm{t} 1)$ and $\operatorname{RST}^{\prime}(\mathrm{i}, \mathrm{j}, \mathrm{t} 1) \neq 0$

- Average degree of spatial dependence: - It is a measure of the extent of similarity of velocities of given two nodes not so far apart, given by $\operatorname{Ds}(i, j, t)$ and averaged over pair of nodes and time instants and formalized by the Equation 7.

$$
D S^{\prime}=\frac{\sum_{i=1}^{T} \sum_{i=1}^{N} \sum_{j=i+1}^{N} D s(i, j, t 1)}{P}
$$

Where $\mathrm{P}$ is no of tuples $(\mathrm{i}, \mathrm{j}, \mathrm{t} 1)$ and $D s(\mathrm{i}, \mathrm{j}, \mathrm{t} 1) \neq 0$

The analysis of mobility models [39] like Steady State Random Waypoint (SSRWMM), Gauss Markov Mobility Model (GMM), City Section Mobility Model (CSMM), Manhattan Grid Mobility Model (MHMM), Reference Point Group Mobility Model (RPGMM) and Realistic Optimized Group Vehicular Mobility Model (ROPGVMM) are illustrated in Figure 2, Figure 3 and Figure 4. 


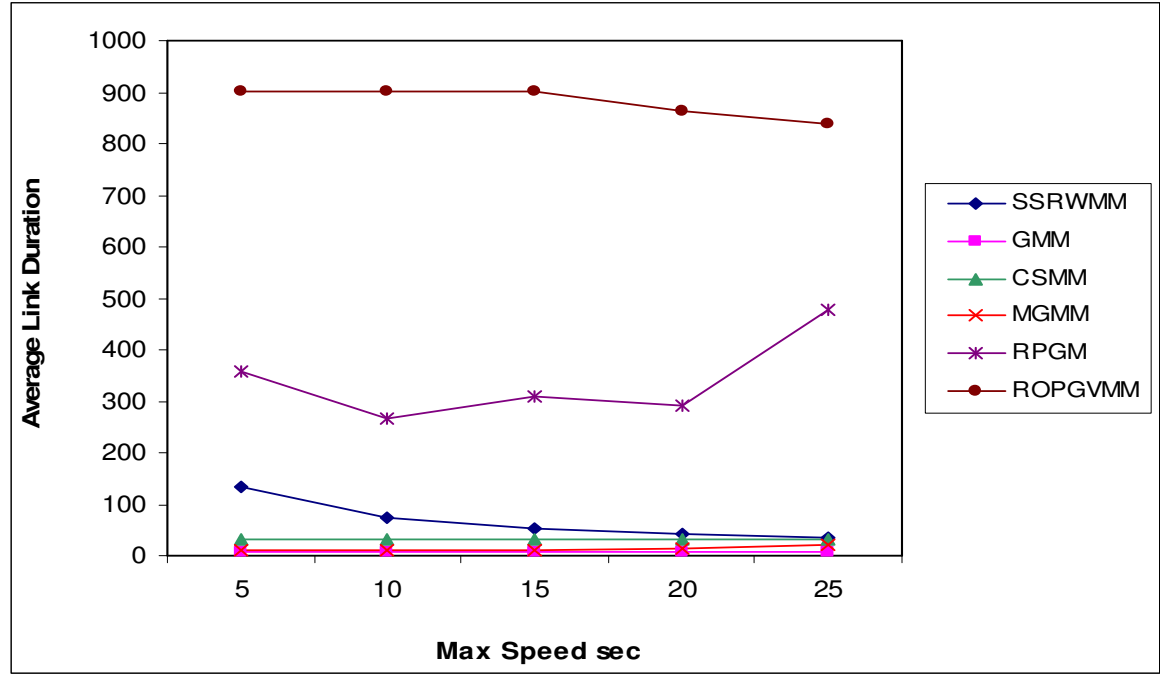

Figure 2. Average Link Duration

In Figure 2 we observe that Manhattan Mobility Model and Gauss Markov Mobility model have the poorest performance relatively as compared to other mobility models. This may possibly be because of high relative speeds exhibited by them and also because of the opposite motion of the nodes in mobility models like Manhattan and City Section. The Random Waypoint Mobility Model with its steady state properties is seen to perform better as compared to Gauss Markov model. The two mobility models which outperform all other mobility models are Reference Point Group Mobility Model and Realistic Optimized Group Vehicular Mobility Model. This is because the nodes in these mobility models deviate by a small percentage from their group leader and their existing links continue to be maintained. This is more relevant in case of Realistic Optimized Group Vehicular Mobility Model where the nodes move in a fixed manner along the streets of a realistic map and hence the better performance. The Realistic Group Vehicular Model outperforms Reference Point Group Model its nearest competitor by $61 \%$

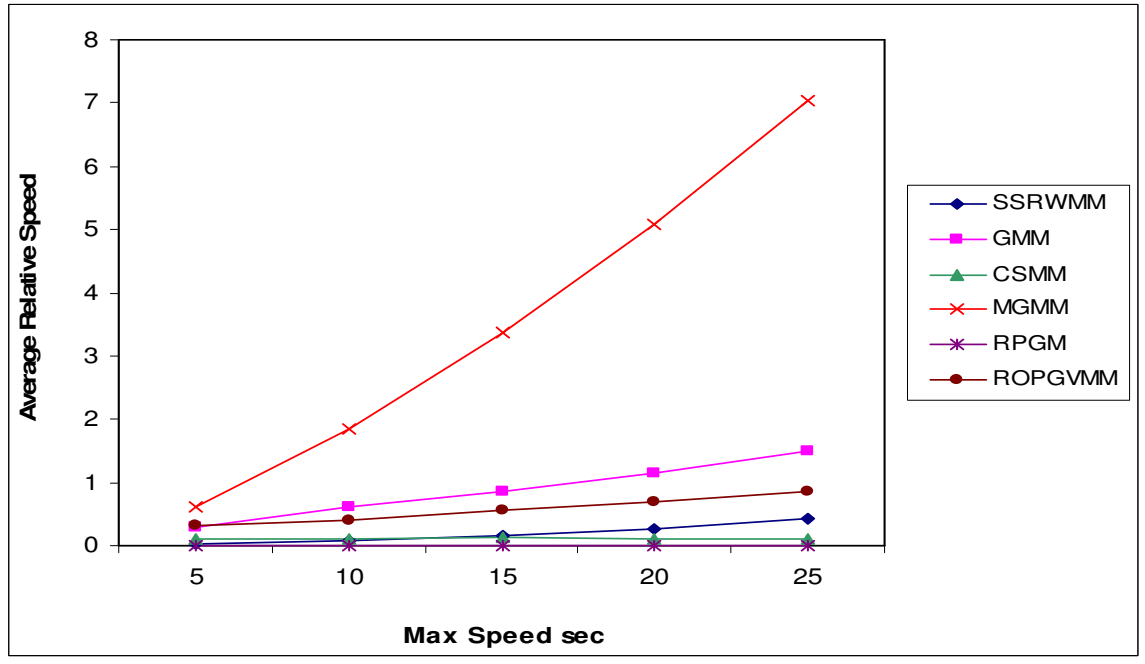

Figure 3. Average Relative Speed 
In Figure 3 it is observed that Manhattan Grid Mobility Model exhibits a high relative speed as compared to other mobility models. This is because of the movement of nodes in opposite direction along the pathways. The average relative speed is least for Reference Point Group Mobility Model since nodes move in co-ordinated fashion along their group leader. The reading of average relative speed is medium for Steady State Random Waypoint Mobility Model. The Realistic Optimized Group Vehicular Mobility Model exhibits a higher average relative speed to the tune of $99 \%$ as compared to Reference Point Group Mobility Model. This may be since that Realistic Optimized Group Vehicular Mobility Model mimics vehicular mobility models and there may be nodes which are moving in opposite direction to cause this effect.

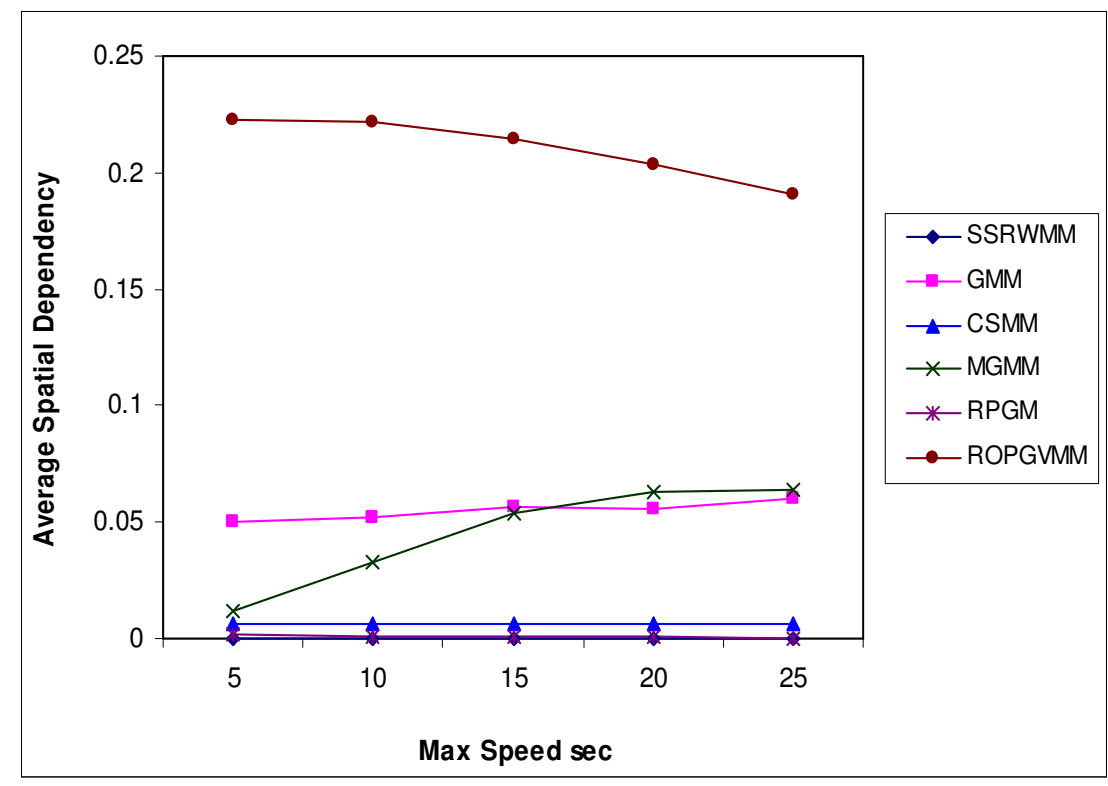

Figure 4. Average Spatial Dependency

It is observed in Figure 4 that Realistic Optimized Group Vehicular Mobility Model outperforms all other mobility models, it also outperforms Reference Point Group Mobility model by $99 \%$. The poor performance of Reference Point Group Mobility Model may be due to the large topology size where nodes may be separated out resulting in network partition and low spatial dependency. Also in case of mobility models which mimic vehicular models we observe that Manhattan Grid Mobility Model outperforms City Section Mobility model. The Steady State Random Waypoint Mobility Model also exhibits very poor performance in case of average spatial dependency.

From the results we observe that Realistic Optimized Group Vehicular Mobility Model outperforms all mobility models. It even outperforms its nearest competitor Reference Point Group Mobility Model. As for our investigation we are characterizing group mobility rather that characterization of individual mobility we consider the two mobility models Realistic Optimized Group Vehicular Mobility Model and Reference point Group Mobility Model. Also these two mobility models have better performance results in terms of mobility metrics considered. 


\section{ROUTING PROTOCOLS IN MANET FOR SCALABILITY}

We consider the two routing protocols AODV and SAMPLE for investigation under scalable environments.

\subsection{Ad-Hoc On-Demand Distance Vector (AODV)}

The AODV [13] is an improvement over DSDV [12] as it is an on-demand routing protocol and strives to minimize the number of broadcasts for route discovery. AODV is considered as a defacto standard for routing under MANET and is thus considered for experimental purposes under scalable environments. The factors that favor AODV under scalable conditions are low overhead, as AODV contains in its packet header only the destination address. Also the route reply packets contain only the destination address and sequence numbers and also contribute to low overheads. Similarly the memory overhead is low as AODV requires knowing only the next hop information to make routing decisions. AODV is among a few routing protocols that supports multicasting. On the other hand the factors which constraint AODV performance under scalable conditions are its requirements for the use of symmetric links, its considers links as discrete in contrast to SAMPLE which considers them as continuous based on historical significance. Furthermore AODV restricts the number of route discovery attempts [35]. This becomes problematic in scenarios where there is less than $1 / 3$ rd success rate resulting in the failure of route discovery process. This problem arises when a critical link serves as a bridge between the two subnetworks, a situation often encountered in vehicular networks.

\subsection{SAMPLE}

To overcome the challenges posed by traditional routing algorithms like AODV [13], DSR [15] and TORA [36] for scalable vehicular network applications for MANET, we consider SAMPLE [17] which is an example of collaborative reinforcement learning routing algorithm. The reason to consider SAMPLE for scalable MANET operations is that it tries to optimize network operations in an adaptive manner. SAMPLE routing protocol is based on generalization of, model based reinforcement learning technique known as Prioritized sweeping [37],[38]. SAMPLE routing protocol does not distinguish between separate control and data messages. This concept proves to be advantageous as only the size of packet is increased in contrast to sending of additional packets in a wireless network. In mobile ad hoc networks where congestion is a norm, some of the links may be down. AODV treats these congested links in a discrete fashion, whereas SAMPLE treats them in a continuous fashion and uses historical data in order to distinguish between a broken links from temporarily congested links.

\subsection{Modelling Reinforcement Learning Based Routing Algorithm for Scalability}

In our investigation of SAMPLE under scalable conditions we consider group mobility for optimization of performance of the routing protocols. Thus we consider two mobility models Reference Point Group Mobility Model (RPGM) and Realistic Optimized Group Mobility Model (ROPGVMM) whose mobility metrics exhibited superior performances as compared to other mobility models. 


\section{Simulation EnVironment for Scalable Conditions}

NS-2 simulator ver. 2.26 from [40] has been used for scalable performance analysis of routing protocols like AODV and SAMPLE. The underlying MAC Protocol is defined by IEEE 802.11. Continuous bit rate (CBR) traffic sources are used. The mobility models used are the Reference Point Group Mobility (RPGM) Model and Realistic Optimized Group Vehicular Mobility (ROPGVMM) Model in a rectangular field. The field configurations are 1920 x $1920 \mathrm{~m}$. The traffic generator script called cbrgen.tcl was used to generate CBR scenario of 15 sources at the rate of $4.0 \mathrm{kbps}$. The number of nodes in the simulation environment was 200 nodes. At least 5 scenarios files for RPGM and 5 scenario files for ROPGVMM at different maximum speed of 5, $10,15,20,25 \mathrm{sec}$ were used for testing protocols like AODV and SAMPLE. We also extend the simulation based studies by varying the number of traffic sources i.e. 15, 20, 25, 30 and 35 at the max speed of $25 \mathrm{sec}$ to test protocol performances under increasing traffic sources at high speed.

\subsection{Routing Protocol Performance Metrics}

The metrics for evaluating the performance of AODV and SAMPLE over Reference Point Group Mobility Model and Realistic Optimized Group Vehicular Mobility Model are as follows as used by Broch [5] for detailed routing protocol performance analysis.

- Packet delivery ratio - The ratio between the numbers of packets originated by the application layer to those delivered to the final destination.

- Path optimality (Average End-End Delay) - The difference between the number of hops a packet took to reach its destination and the length of the shortest path that physically existed through the network when the packet was originated.

The packet delivery ratio and path optimality metrics are important for best-effort traffic. The routing overhead metric evaluates the efficiency of the routing protocol. We could not apply routing overhead metric as SAMPLE does not distinguish between data and routing messages.

\subsection{Scalable Simulation results}

The results after simulating SAMPLE and AODV under scalable conditions are illustrated in Figure 5 (a), Figure 5 (b) and Figure 6(a) and Figure 6(b). 
International Journal of Computer Networks \& Communications (IJCNC) Vol.2, No.6, November 2010

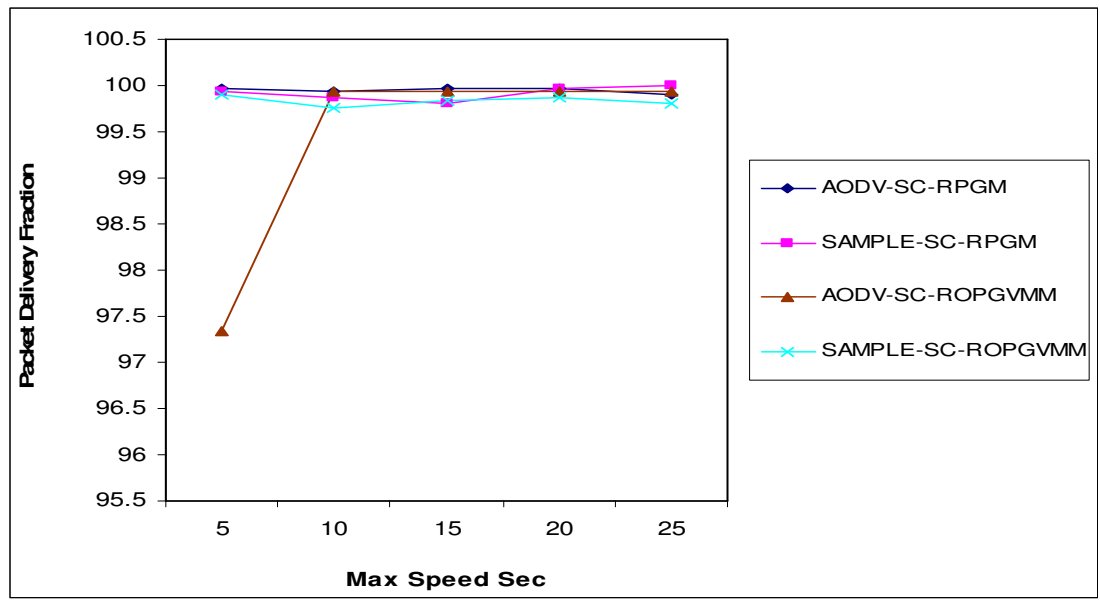

Figure 5 (a) Packet Delivery Fraction for AODV and SAMPLE for scalable conditions under varying max speed

In Figure 5(a) we observe that both the routing protocols AODV and SAMPLE have relatively optimal performances. Also the reason for improved performances was that the mobility metrics for RPGM and ROPGVMM were superior in terms of average link duration. This has contributed to superior performances in realistic city scenario where there may be a possibility of nodes moving in opposite direction. The high level of spatial dependency exhibited by ROPGVMM where nodes move in cluster around the group leader has contributed towards the superior performance of reinforcement based learning algorithm SAMPLE and its competitor AODV. In case of ROPGVMM, AODV initially has relatively low packet delivery fraction and then settles to ideal scenario. The performances of packet delivery fraction which indicates the robustness of a routing protocol is similar on both the mobility models.

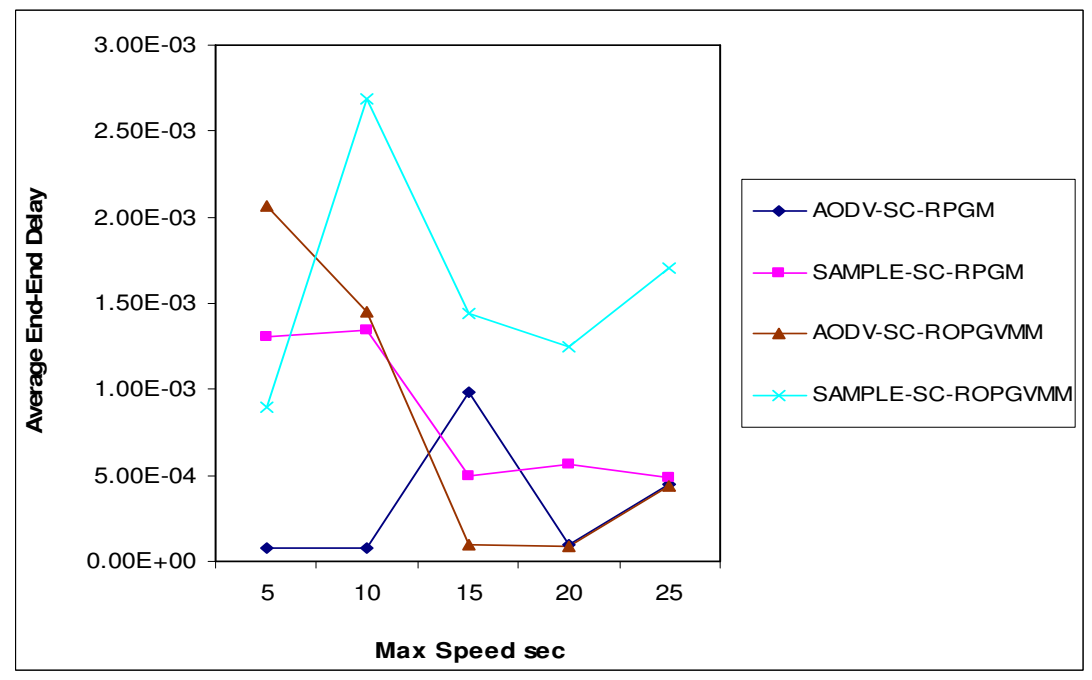

Figure 5(b) Average End-End Delay for AODV and SAMPLE for scalable conditions under varying max speed 
In Figure 5(b) we observe that the average end-end delay for SAMPLE routing protocol is relatively inferior on both mobility models RPGM and ROPGVMM. AODV prefers least congested routes by replying to the first route request packet and does not consider the route length. Thus we observe that AODV exhibits lower delay on both mobility models. SAMPLE on the other hand tries to balance route discovery versus exploitation and thus sometimes may have to choose suboptimal paths resulting in a low average end-end delay.

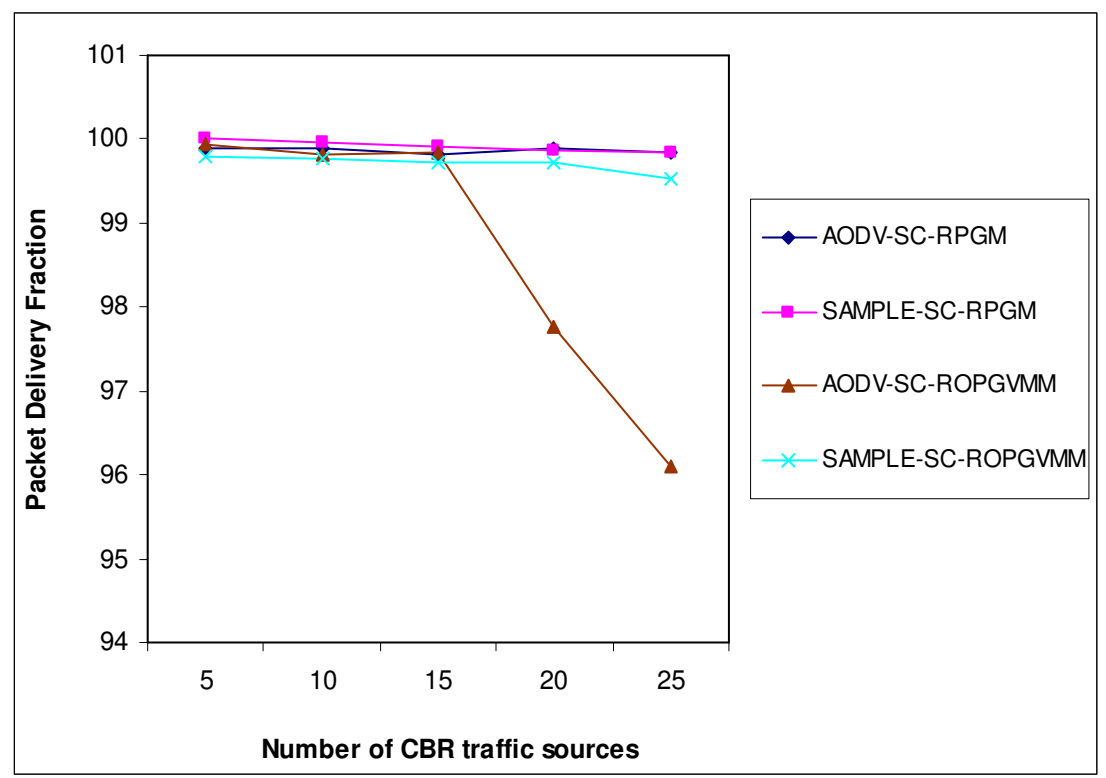

Figure 6(a) Packet Delivery Fraction for AODV and SAMPLE for scalable conditions under varying traffic sources

In Figure 6(a) we extend our simulation based studies under varying traffic conditions. We observe that the performances of SAMPLE on ROPGVMM match the performances of AODV on RPGM in terms of packet delivery fraction. However the performance of AODV dips as the rate of cbr sources increases on the ROPGVMM. The performance of SAMPLE on ROPGVMM remains stable and outscores AODV under 20 and 25 cbr sources by $4 \%$. Thus we can observe that under scalable conditions under varying traffic and high speed conditions, SAMPLE protocol maintains a stable performance and slightly outperforms AODV protocol under specific scenarios of 20 and 25 cbr sources. 


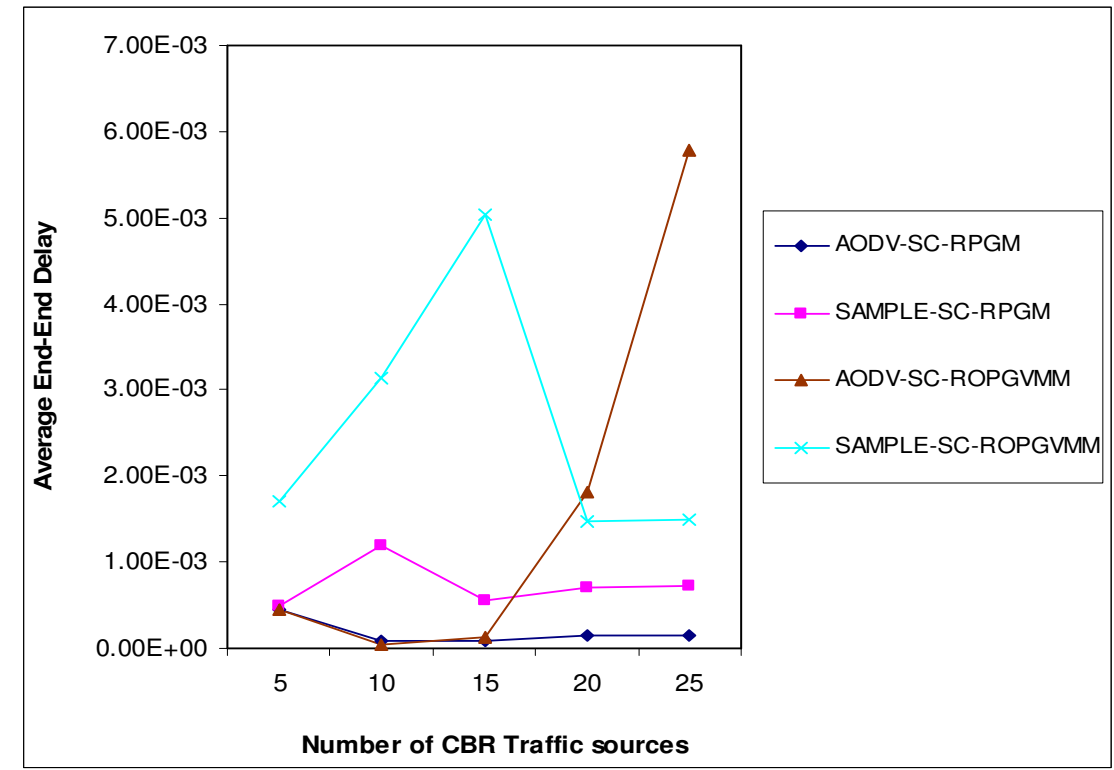

Figure 6(b) Average End-End Delay for AODV and SAMPLE for scalable conditions under varying traffic sources

In Figure 6(b) we observe that under RPGM mobility model AODV outperforms SAMPLE routing protocol in terms of average end-end delay. In case of the ROPGVMM we observe that AODV for low number of traffic sources outscores SAMPLE. But as the number of CBR sources increases the average end-end delay stabilizes for SAMPLE, but is seen to grow exponentially for AODV. Thus on the realistic model ROPGVMM, which we have proposed, SAMPLE end-end delay metric stabilizes under scalable conditions with high speed and varying traffic conditions

\section{Conclusion}

For any type of network to be popular it must exhibit scale of economies. Thus in our endeavour we emphasized on scalable applications for mobile ad hoc networks. We consider that mobility models play a vital role in the dynamic of ad hoc networks as they mimic the real world. Most of the research in ad hoc networks has been towards the individual characterization of mobile node. In our work we emphasized on group characterization of mobile nodes. We also proposed a new mobility model which extends the important characteristics of group mobility and applies it to vehicular motion of mobile nodes derived from realistic maps. This mobility model would help us to visualize the scalable application of MANET. In our analysis of mobility models on various metrics, it was observed that the two group mobility models Reference Point Group Mobility Model (RPGM) and Realistic Optimized Group Vehicular Mobility Model (ROPGVMM) performed relatively better as compared to the other mobility models. Thus for further experimentation both RPGM and ROPGVMM were considered. As routing protocols are central to the design of ad hoc networks, we considered two routing protocols namely AODV and SAMPLE. AODV is the de facto standard for routing in MANETs and has proven capabilities under varying environments. SAMPLE is an example of reinforcement learning algorithm and was our main focus of investigation to achieve its optimal performances under scalable environments. In scalable conditions both protocol performed similar in terms of packet delivery 
fraction. The average end-end delay of SAMPLE exhibited slightly poor performance; however under varying traffic conditions it exhibited more stable performance. Thus we can further our work on a reinforcement learning approach as a routing solution for a scalable realistic applications of mobile ad hoc networks.

\section{REFERENCES}

[1]. Tavli B. and Heinzeiman W (2006) Mobile Ad Hoc Networks- Energy Efficient Real-Time Data Communication, Springer pp. 2 - 5.

[2]. Corson S and Marker J (1999). Mobile Ad Hoc Networking (MANET): Routing Protocol Performance Issues and Evaluation Considerations, RFC 2501.

[3]. Yoon J, Liu M and Noble B (2003). Random Waypoint Considered Harmful, Proceedings of IEEE INFOCOM 2003, San Francisco, C A.

[4]. Johnson D B and Maltz D.A (1996). Dynamic source routing in ad hoc wireless networks in mobile computing. In Imielinkski and Korth. (Eds), Kluwner Academic Press.

[5]. Broch J, Maltz D.A, Johnson D.B, Hu Y-C and Jetcheva J (1998). A performance comparison of multi-hop wireless ad hoc network routing protocols. Mobile Computing and Networking (MobiCom) pp. 85-97.

[6]. Chaudri S P, Boudec JYL, Vojnovic M (2005). Perfect Simulations for Random Trip Mobility Models. Proceedings of the $38^{\text {th }}$ Annual Symposium on Simulations, pp. 72-79.

[7]. UMTS 30.03 Version 3.2.0 (1998). ETSI, Selection procedures for choice of radio transmission technologies of the UMTS Universal Mobile Telecommunications System (UMTS) , http://www.3gpp.org/ftp/specs/html-info/ 3003u.htm

[8]. Bonn Motion (2005). A mobility scenario generation and analysis tool http://web.informatik.unibonn.de/iv/mitarbeiter/decvaal/bonnmotion

[9]. Davies V (2000). Evaluating Mobility Models within an Ad Hoc Network, Master Thesis, Colorado School of Mines.

[10]. Walrand J, Varaiya P (2005). High Performance Communication Networks. Morgan Kaufman, San Francisco.

[11]. Gerla M (2005). Ad Hoc Networks Emerging Applications, Design Challenges and Future Opportunities in Mohapatra P and Krishnamurthy S (Eds). Ad Hoc Networks Technologies and Protocols, Springer Boston.

[12]. Perkins $C$ and Bhagwat P (1994). Highly dynamic destination-sequenced distance-vector routing (DSDV) for mobile computers. Proceedings of the ACM SIGCOMM, pp. 234-244.

[13]. Perkins C and Royer E (1999). Ad hoc on-demand Distance Vector Routing. Proceedings of the $2^{\text {nd }}$ IEEE Workshop on Mobile Computing Systems and Applications, Feb, pp. 90-100.

[14]. Perkins C, Royer E and Das S. Ad Hoc on demand distance vector (AODV) routing, http://www.ietf.org/internet-drafts/draft-ietf-manet-aodv-03.txt

[15]. Broch J, Johnson D and Maltz D. The dynamic source routing protocol for Mobile ad hoc networks, http://www.ietf.org/internet-drafts/draft-ietf-manet-dsr-0.1.txt

[16]. Ethem A (2006). Introduction to Machine Learning. PHI, New-Delhi, India.

[17]. Dowling J, Curran E, Cunningham R and Cahill V (2005). Using Feedback in Collaborative Reinforcement Learning to Adaptively Optimize MANET Routing. IEEE Transactions On Systems Man and Cybernetics. Part A: Systems and Humans, Vol.3. 
International Journal of Computer Networks \& Communications (IJCNC) Vol.2, No.6, November 2010

[18]. TIGER (2003) Topologically Integrated Geographic Encoding and Referencing. http://www.census.gov/geo/www/tiger/tiger2003/tgr2003.html.

[19]. Nurmi P (2007). Reinforcement Learning for Routing in Ad Hoc Networks. Proceedings of 5th Intl. Symposium on Modeling and Optimization in Mobile, Ad Hoc, and Wireless Networks (WiOpt, IEEE Computer Society Limassol, Cyprus.

[20]. Kwak B-J, Nah-Oak and Leonard E M (2004).On the Scalability of Ad Hoc Networks: a traffic analysis at the center of a network. Proceedings of IEEE WCNC 2004, Atlanta.

[21]. Valery Naumov, Thomas Gross, "Scalability of routing methods in ad hoc networks", In Performance Evaluation Journal (Elsevier Science), Volume 62, pp 193-207.

[22]. Bai F, Sadagopan N and A Helmy A (2003). The IMPORTANT framework for analyzing the Impact of Mobility on Performance of RouTing protocols for Adhoc NeTworks. Elsevier Ad Hoc Networks, 1, 383-403.

[23]. Zhou B, Xu K, and Gerla M (2004). Group and Swarm Mobility Model for Ad Hoc Networks Scenarios Using Virtual Tracks. IEEE Military Communications Conference (MILCOM), Monterey, CA, USA.

[24]. Tewari N A, Das S, Gerla M, and Kleinrock L (2006). Adtorrent: Delivering Location Cognizant Advertisements to Car Networks. IEEE/IFIP Annual Conference on Wireless On-Demand Network Systems and Services (WONS), Les Menuires, France.

[25]. Hong X, Gerla M, Pei G and Chiang C-C (1999). A Group Mobility Model for Ad Hoc Wireless Networks. ACM/IEEE International Symposium on Modeling Analysis and Simulation of Wireless and Mobile Systems (MSWiM), Seattle, WA, USA.

[26]. Sarkar S K, Basavaraju T G and Puttamadappa C (2008), Ad Hoc Mobile Wireless Networks, Auerbach Publications, Boca Raton, FL, pp. 261-270.

[27]. Kurkowski S, Camp T and Navidi W. Minimal Standards for Rigorous MANET Routing Protocol Evaluation, Technical Report MCS 06-02, Colorado School of Mines.

[28]. Boleng J( 2001), Normalizing mobility characteristics and enabling adaptive protocols for ad hoc networks, Proceedings of the Local and Metropolitan Area Networks Workshop (LANMAN), pp. 912.

[29]. Boudec J-Y L and Vojnovi'c M (2005). Perfect simulation and stationarity of a class of mobility models. Proceedings of IEEE Infocom 2005.

[30]. Camp T, Boleng J and Davies V (2002). A survey of Mobility Models for Ad Hoc Network Research. http://toilers.mins.edu/papers/psgz/models.ps.gz.

[31]. Liang, Haas B and Zygmunt J (1999). Predictive distance based mobility management for a PCS Networks. Proceedings of the IEEE Infocom.

[32]. Saha A K, Johnson D B (2004). Modeling Mobility for Vehicular Ad Hoc Networks. Proceedings of the 1st ACM international workshop on Vehicular ad hoc networks, Philadelphia, PA, USA, pp. 9192.

[33]. PalChaudhuri S. ns-2 Code for Random Trip Mobility Model, http://monarch.s.rice.edu/ santa/research /mobility/

[34]. Bai F, Sadagopan N, Helmy A. IMPORTANT: An evaluation framework to study the "Impact of Mobility Patterns On RouTing in Ad-hoc NeTworks", http://nile.cise.ufl.edu/important/

[35]. Ros F J, Ruiz P M, Sanchez J A and Stojmenovic I (2009). Mobile Ad Hoc Routing in Context of Vehicular Networks in Solariu, MC Weigle (Eds). Vehicular Networks From Theory To Practise, pp 9-19 to 9-21CRC Press, Boca Raton, FL. 
[36]. Park V and Corson M. (1997). A highly adaptive distributed routing algorithm for mobile wireless networks. Proceedings of the IEEE INFOCOM, pp. 1405-1413.

[37]. Moore A W, Atkeson C G (1993). Prioritized Sweeping: Reinforcement Learning with Less data and Less Time. Machine Learning.

[38]. Nash K. (2005). Implementation of SAMPLE protocol, Dissertation Report, University of Dublin, Trinity College.

[39]. Curran E (2004), SWARM: Cooperative Reinforcement Learning for Routing in Ad Hoc Networks, M.S. Thesis, Trinity College Dublin.

[40]. The Network Simulator - NS-2, http://www.isi.edu/nsam/ns/

\section{Authors}

Shrirang Ambaji Kulkarni is working as Assistant Professor in the Department of Computer Science and Engineering, Gogte Institute of Technology, Belgaum. He received his B.E. in Computer Science and Engineering from Karnataka University Dharwar, in the year 2000 and his M.Tech. in Computer Network Engineering from VTU Belgaum, in the year 2004. He is currently pursuing Ph.D. in the area of ad hoc networks at N.I.E Mysore. He has published more than 10 papers in International, National Journals and International conferences. His current research interest includes Reinforcement Learning Algorithms and Wireless Ad Hoc Networks .He is a life member of CSI and ISTE.

Dr. G. Raghavendra. Rao received his Masters in Engineering from Indian Institute of Science Bangalore in the year 1987 and his Ph.D. from University of Mysore in the year 1999. He is currently working as Professor in Department of Computer Science and Engineering at NIE Mysore. He has published more than 25 papers in International, National Journals and International Conferences His current research interest includes Genetic Algorithms, Wireless Networks, Cryptography and Network Security. He is a member of IEEE, ACM and ISTE.
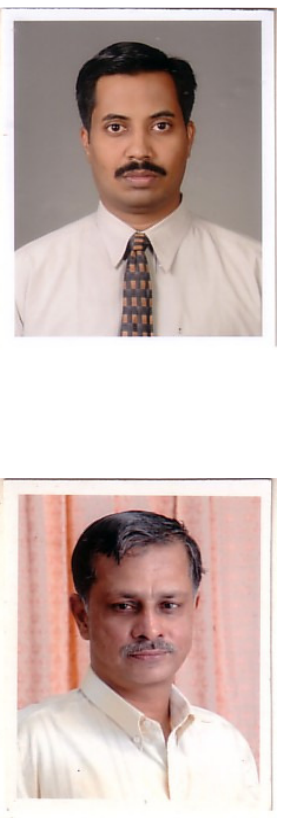